\title{
Blockade of miR-663b inhibits cell proliferation and induces apoptosis in osteosarcoma via regulating TP73 expression
}

\author{
Shu $\mathrm{Y}^{1}, \mathrm{Ye} \mathrm{W}^{2}, \mathrm{Gu} \mathrm{YL}^{3}$, Sun $\mathrm{P}^{4}$ \\ Department of Orthopedics, The Sixth Hospital of Wuhan, Affiliated Hospital of Jianghan University, \\ WuHan, Hubei Province, China.guyl201708@163.com
}

\begin{abstract}
OBJECTIVE: This study aimed to investigate the exact role of miR-663b in osteosarcoma (OS) progression and further explore the underlying molecular mechanisms.

MATERIALS AND METHODS: The miR-663b expression in human OS cell lines was determined by qRT-PCR, and the results suggested that miR-663b was highly expressed in human OS cells. TargetScan was used to predict the potential targets of miR-663b, and the prediction was confirmed by dual-luciferase reporter assay. To investigate the role of miR-663b in OS, miR-663b was down-regulated in U2OS cells using miR-663b inhibitor. CCK8 and flow cytometry were preformed to investigate the proliferation and apoptosis of U2OS cells. Moreover, qRT-PCR and western blot analysis were performed to measure the mRNA and protein expression. RESULTS: We found that miR-663b directly targets TP73 and negatively regulates TP73 expression. MiR-663b inhibitor significantly decreased the proliferation ability of U2OS cells, while the percentage of apoptotic cells was markedly increased. The level of Bcl-2 was notably inhibited by miR-663b inhibitor, while Bax expression was significantly enhanced. Moreover, miR-663b down-regulation promoted p53 and p21 expression in U2OS cells. CONCLUSIONS: MiR-663b down-regulation represses proliferation and induces apoptosis in OS by targeting TP73. Therefore, we provide a potential therapeutic target for OS treatment (Fig. 6, Ref. 27). Text in PDF www.elis.sk. KEY WORDS: osteosarcoma, miR-663b, TP73, proliferation, apoptosis.
\end{abstract}

\section{Introduction}

Osteosarcoma (OS), the primary malignant bone tumor in young adults and children, is frequently derived from long bones and characterized by osteoid production produced by malignant cells (1-5). OS has high mobility and aggressivity, mainly to the lung. At present, the main therapeutic mean for OS is the combination treatment with chemotherapy and surgery, however, the five-year survival of OS patients remains relatively low $(6,7)$. Therefore, it is urgent for researchers to explore new therapeutic targets for OS treatment.

MicroRNAs (miRNAs), a class of short non-coding RNAs, 18-22 nucleotides in length, can negatively regulate the expres-

${ }^{1}$ Department of Orthopedics, The Sixth Hospital of Wuhan, Affiliated Hospital of Jianghan University, WuHan, Hubei Province, China, ${ }^{2}$ Department of Neonatology, Renmin Hospital of WuHan University, WuHan, Hubei Province, China, ${ }^{3}$ Department of Orthopedics, Wuxi Second Hospital, Nanjing Medical University, Wuxi, Jiangsu Province, China, and ${ }^{4}$ Department of Pathology, Wuxi Second Hospital, Nanjing Medical University, Wuxi, Jiangsu Province, China

Address for correspondence: Y.L. Gu, No. 68 Zhongshan Road, Wuxi 214002, Jiangsu province, China.

Phone: +86.0510 .66651691 , Fax: +86.0510 .68562142$

Acknowledgements: This study was supported by the Jiangsu Top Six Talent Foundation, China (WSW099), Jiangsu health young talent Foundation (QNRC2016150), Wuxi Health and Family Planning Commission youth foundation (Q201407) and Wuxi Health and Family Planning Commission Key Foundation (YGZXZ1503). sion of the target genes via binding to the 3'-untranslated regions (UTR) of the specific gene mRNAs (8). MiRNAs play an important role in regulating a variety of biological processes, including cell proliferation, cell cycle, apoptosis and differentiation (9-11). Evidences have indicated that miRNAs play critical roles in managing many normal biological processes, and abnormal expression of miRNAs is associated with various cancers occurrence, such as gastric cancer, lung cancer, OS, etc. (12-14), and miRNAs serve as oncogenes or cancer suppressors during the progression of OS $(15$, 16). For example, Bi et al (17) suggested that miR-100 serves as a cancer inhibitor in OS development via suppressing OS growth by regulating FGFR3 expression. MicroRNA-133a can prevent the proliferation and invasion of osteosarcoma cells by targeting IGF$1 \mathrm{R}$ (18). MiR-542-5p has been found to serve as an oncogene in OS though promoting OS proliferation by targeting HUWE1 (19).

MiR-663b has been found to be up-regulated in OS (14), however, the exact roles of miR-663b in OS and the underlying molecular mechanisms are still unclear. Thus, in this study, we will investigate the role of miR-663b in OS and further explore the underlying molecular mechanisms.

\section{Materials and methods}

\section{Materials}

The normal osteoblast hFOB1.19 cell line and human osteosarcoma (HOS) cell lines MG63 and U2OS were obtained from American type culture collection (ATCC); the RPMI 1640 me- 
dium, DMEM medium and fetal bovine serum (FBS) were obtained from Invitrogen company (Waltham, MA, USA); the primary antibodies and the second antibody were supplied by Cell Signaling Technology company (Danvers, MA, USA); the CCK8 kit was purchased from Eli Lilly and Company (USA); and the lipofectamine 2000 cell transfection reagent was purchased from Invitrogen company (USA).

\section{Cell culture}

The U2OS cells were grown in RPMI 1640 medium containing $10 \%$ fetal bovine serum (FBS), $1 \%$ penicillin-streptomycin (Invitrogen), and incubated in humidified atmosphere of $5 \% \mathrm{CO}_{2}$ at $37^{\circ} \mathrm{C}$. The MG63 cells were cultured in DMEM medium. The normal osteoblast hFOB1.19 cell line was grown in F12/DMEM (v/v: 1: 1, Hyclone) medium containing $10 \% \mathrm{FBS}, 2.5 \mathrm{mM} / \mathrm{L}$ glutamine (without phenol red), and $0.3 \mathrm{mg} / \mathrm{ml} \mathrm{G418} \mathrm{(Sigma,} \mathrm{St.} \mathrm{Louis,} \mathrm{MO,}$ USA). Fresh cell culture medium was replaced every 2-3 days.

\section{Dual luciferase reporter assay}

TargetScan was used in the present study to predict the targets of miR- $663 \mathrm{~b}$, and TP73 was found to be a potential target of miR$663 \mathrm{~b}$. To reveal the prediction, dual luciferase reporter assay was applied. Wild-type and mutant 3'-UTRs of TP73 were amplified and then cloned into the psiCHECK-2 reporter. MiR-663b and miR-663b-TP73-WT 3'UTR or miR-663b-TP73-MUT 3'UTR vector were co-transfected into U2OS cells using Lipofectamine 2000 regent following the instructions provided by the manufacturer. $48 \mathrm{~h}$ later, the luciferase activity was assessed by using the Dual-Luciferase Reporter Assay System (Promega, USA).

\section{Cell transfection}

To investigate the role of miR-663b in OS, a stable miR-663blow expression U2OS cell line was generated via performing cell transfection assay. In brief, U2OS cells were transfected with miR$663 \mathrm{~b}$ inhibitor, its negative control, or miR-663b inhibitor + siTP73 RNA by using Lipofectamine 2000 (Invitrogen, USA) according to the manufacturer's instructions. $48 \mathrm{~h}$ after the cell transfection, the U2OS cells were collected and then used for subsequent analysis.

\section{Cell proliferation assay}

$48 \mathrm{~h}$ after transfection, the U2OS cells were seeded into a 96well culture plate (Corning Costar, Corning, NY, USA) $(2,500$ cells per well) and then incubated for $24 \mathrm{~h}$ at $37{ }^{\circ} \mathrm{C}$ under $5 \%$ (v/v) $\mathrm{CO}_{2}$. Subsequently, $10 \mu \mathrm{g} / \mathrm{ml} \mathrm{CCK} 8$ solution was added to each well at certain time points, and then incubated at $37^{\circ} \mathrm{C}$ for 2 $\mathrm{h}$. At the end of the test, the OD value at $450 \mathrm{~nm}$ was detected by using a microplate reader (Thermo Labsystems, Waltham, MA, USA). Experiments were repeated at least 3 times.

\section{Apoptosis analysis assay}

$48 \mathrm{~h}$ after transfection, cells were washed for at least 3 times using cold PBS solution. Then the U2OS cells were fixed with 70 $\%$ ethanol (30 $\mathrm{min})$, rinsed with PBS solution, labeled with annexin V-FITC and propidium iodide (PI), and then incubated at room temperature for $30 \mathrm{~min}$. Finally, cell apoptosis was analyzed us- ing the flow cytometry (Becton Dickinson, New Jersey, USA) and cell apoptotic rate was calculated. Tests were repeated three times.

\section{Western blot}

$48 \mathrm{~h}$ after cell transfection, log-phase U2OS cells were harvested, and total cellular protein was extracted by using RIPA Buffer (Auragene, Changsha, China). The BCA protein quantitative kit (Thermo, USA) was applied to determine protein concentration. Equal amount of protein samples were resolved by using SDSPAGE analysis and then transferred to a polyvinylidene fluoride (PVDF) membrane. The membrane was then blocked with $5 \%$ fat-free milk at room temperature for $1 \mathrm{~h}$. Subsequently, the membrane was incubated with a primary antibody (anti- $\beta$-actin $1: 1000$; anti-p53 1: 1000; anti-p21 1: 1000; anti-TP73 1: 1000; anti-Bcl-2 $1: 1000$; anti-Bax $1: 1000$ ) at $4{ }^{\circ} \mathrm{C}$ overnight, then washed three times with TBST solution, followed by incubation with a HRPconjugated secondary antibody (Anti-rabbit IgG, HRP-linked Antibody; $1: 5,000$ ) at room temperature for $1 \mathrm{~h}$. To visualize the protein bands, an ECL kit (Applygen, Beijing, China) was applied following the manufacturer's protocol.

\section{QRT-PCR}

Total RNA of U2OS cells was extracted using TRIZOL reagent (Takara, Japan) according to the instructions supplied by the manufacturer. To evaluate the quality and integrity of the RNA, A260/A280 ratios were assessed. U6 and GAPDH served as internal controls for the miRNA and mRNA expression, respectively. The PrimeScript ${ }^{\mathrm{TM}}$ RT reagent kit (Takara, Japan) was used for cDNA generation. SYBR Premix Ex Taq (Takara, Japan) was carried out for RT-PCR analysis. The TaqMan MicroRNA Reverse Transcription Kit (Life Technologies, USA) was used to reversely transcribe miRNA into cDNA, and RT-PCR was carried out by using the SYBR RT-PCR kit (Takara, Japan).

\section{Statistical analysis}

All quantitative data are shown as the mean \pm SD. SPSS 17.0 statistical software (SPSS, Chicago, IL, United States) was applied for statistical analyses. Student's t-test or ANOVA was used to compare differences between groups. $\mathrm{p}<0.05$ was considered statistically significant.

\section{Results}

miR-663b is up-regulated in osteosarcoma cell lines

The expression level of miR-663b was determined by qRTPCR in the human osteosarcoma MG-63, U2OS and osteoblast hFOB1.19 cell lines. The results suggested that compared with the control, the expression of miR-663b was significantly increased in MG-63 and U2OS cells, especially in U2OS cells, indicating that miR-663b was up-regulated in OS (Fig. 1). U2OS cells were used for further analysis.

\section{$P 73$ is a target of $\mathrm{miR}-663 \mathrm{~b}$}

To investigate the role of miR-663b in the development of OS, TargetScan was performed to predict the target gene of miR-663b 


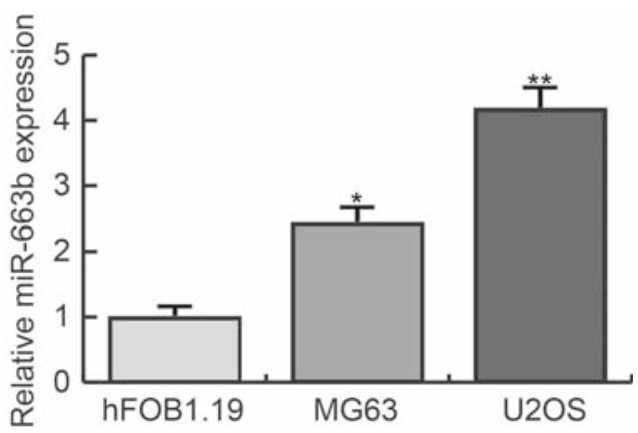

Fig. 1. miR-663b is up-regulated in osteosarcoma cell lines. We performed qRT-PCR to determine the miR-663b expression. hFOB1.19: normal human osteoblastic cell line; MG63 and U2OS: human osteosarcoma cell lines. ${ }^{*}, *$ p $<0.05,0.01$ vs hFOB1.19. Experiments were repeated 3 times.

(Fig. 2A), and the luciferase reporter gene assay was carried out to confirm our prediction. The results showed that the luciferase activity was significantly reduced in miR-663b and miR-663bTP73-WT co-transfected U2OS cells, but co-transfection of miR$663 \mathrm{~b}$ with miR-663b-TP73-MUT did not reduce it (Fig. 2B). These results revealed miR-663b could inhibit expression of transcripts containing a miR-663b binding site, indicating that TP73 is a direct target gene of miR-663b.

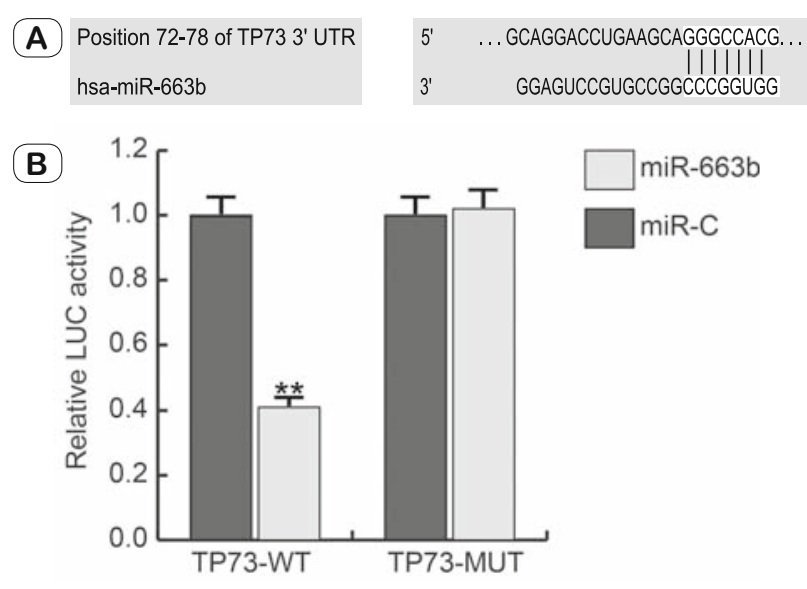

Fig. 2. TP73 is a direct target of miR-663b. A: Interaction between miR-663b and 3'UTR of TP73 was predicted using TargetScan; B: Luciferase activity of a reporter containing a wild-type TP73 3'UTR or a mutant TP73 3' UTR are presented. “TP73-3'UTR-MUT” indicates the TP73 3' UTR with a mutation in the miR-663b binding site. UTR, untranslated region. ${ }^{* *} \mathrm{p}<0.01$ vs control. All data are presented as the mean \pm SD of three independent experiments.

Furthermore, we found that TP73 had low expression in U2OS cells (Figs 3A and B). Moreover, to determine the role of miR$663 \mathrm{~b}$ in U2OS cells, U2OS cells were transfected with miR-663b inhibitor, its negative control, or miR-663b inhibitor + siTP73

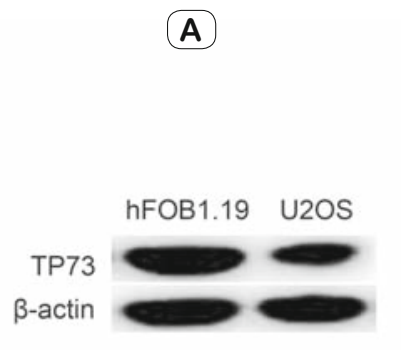

(D)

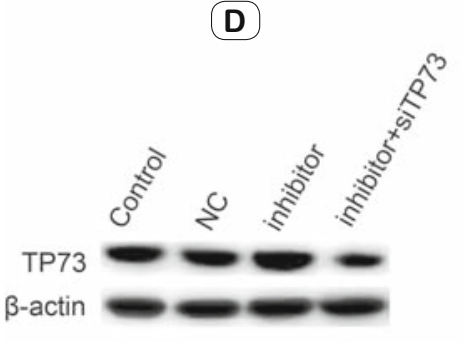

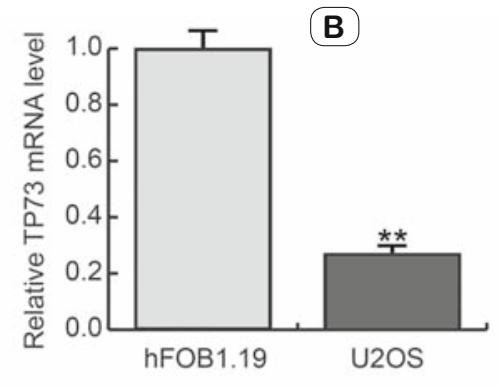

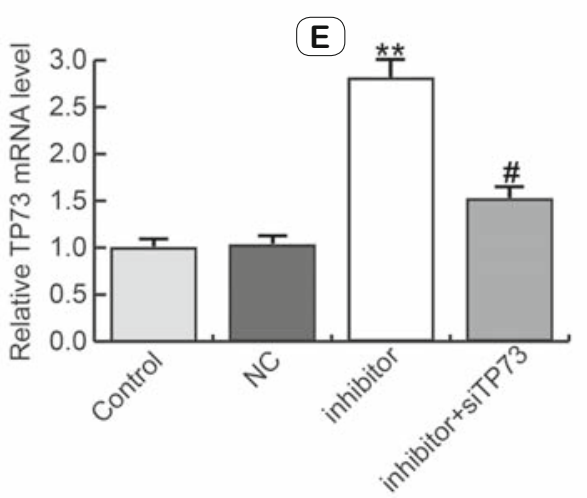

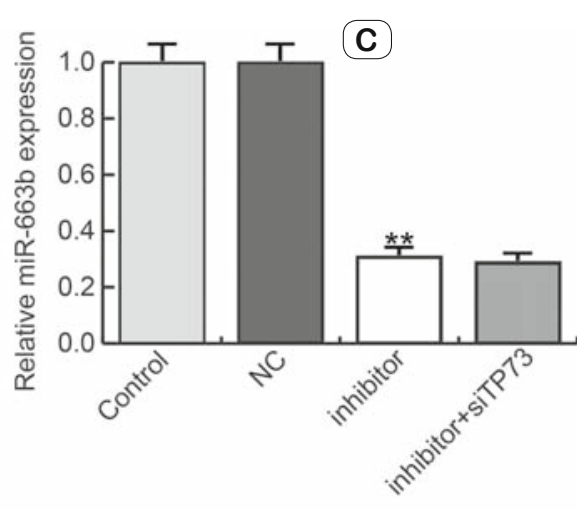

Fig. 3. Expression of TP73 in different groups. A: protein level of TP73 in hFOB1.19 and U2OS cell line was measured by western blotting; B: mRNA level of TP73 in hFOB1.19 and U2OS cell lines were measured by qRT-PCR; C: relative miR-663b expression in different groups was detected by qRT-PCR; D: protein level of TP73 in different groups was detected by western blotting; E: mRNA level of TP73 in different groups was detected by qRT-PCR. Control: cells without any treatment; NC: cells transfected with the negative control of miR-663b inhibitor; inhibitor: cells transfected with miR-663b inhibitor; inhibitor+siTP73: cells co-transfected with miR-663b inhibitor and siTP73 RNA. All data are presented as the mean \pm SD of three independent experiments. ${ }^{* *} \mathbf{p}<0.01$ vs control; \# $\mathbf{p}<0.05$ vs inhibitor. 


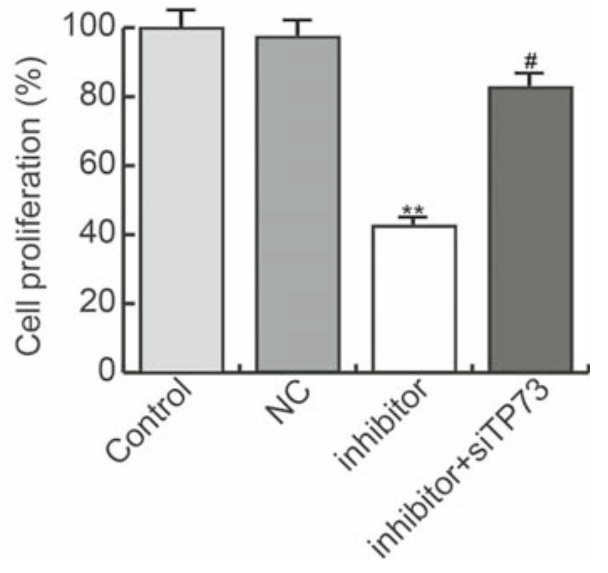

Fig. 4. miR-663b down-regulation inhibits the proliferation of U2OS cells. Cell proliferation was analyzed by CCK8 assay in the U2OS cells. Control: cells without any treatment; NC: cells transfected with the negative control of miR-663b inhibitor; inhibitor: cells transfected with miR-663b inhibitor; inhibitor+siTP73: cells co-transfected with miR-663b inhibitor and siTP73 RNA. All data are presented as the mean \pm SD of three independent experiments. ${ }^{* *} p<0.01$ vs control; $\# \mathbf{p}<0.05$ vs inhibitor.

RNA, and the transfection efficiency was assessed by qRT-PCR (Figure 3C). And we found that miR-663b negatively regulated TP73 expression in U2OS cells. miR-663b inhibitor significantly increased TP73 expression, and this increase could be eliminated by siTP73 RNA (Figs 3D and E).

miR-663b down-regulation suppresses the proliferation of U2OS cells

To explore the effect of miR-663b on OS cell proliferation, cell proliferation was measured by using CCK-8 assay. As shown in Figure 3, miR-663b inhibitor markedly decreased the proliferation ability of U2OS cells, and this decrease could be eliminated by siTP73 RNA (Fig. 4). The data indicated that miR-663b down-regulation inhibited the proliferation of osteosarcoma cells.

miR-663b down-regulation induces the apoptosis of osteosarcoma cells

To investigate whether cell apoptosis participated in the proliferation repression caused by miR-663b inhibitor apoptosis of the U2OS cells was analyzed by using flow cytometry. As shown in Figures 5A and B, compared with the control, miR-663b downregulation notably increased the apoptotic rate of $\mathrm{U} 2 \mathrm{OS}$ cells, and this increase could be eliminated by siTP73 RNA.

In addition, the expression level of Bcl-2 and Bax was determined, and we found that miR-663b inhibitor significantly decreased Bcl-2 expression, and the expression level of Bax was markedly enhanced. These effects could be offset by siTP73 RNA (Fig. 5C).

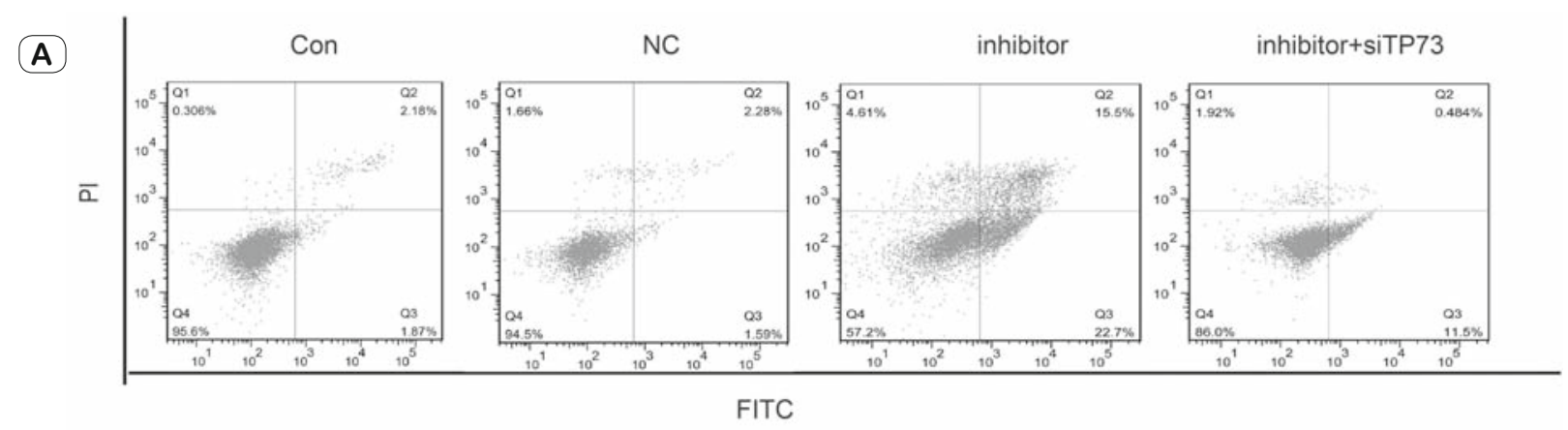

B

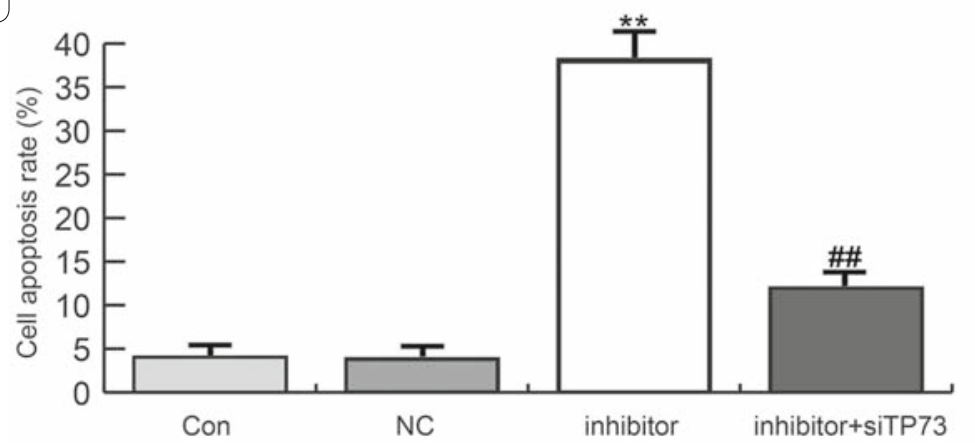

C

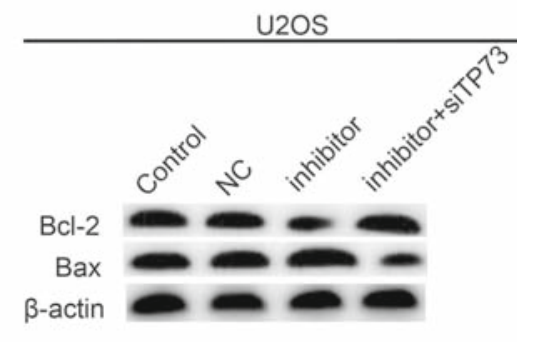

Fig. 5. miR-663b down-regulation induces the apoptosis of U2OS cells. Flow cytometry was performed to detect the cell apoptosis (A), and data was analyzed (B). C: Bax and Bcl-2 protein level in different groups were detected by western blotting. Control: cells without any treatment; NC: cells transfected with the negative control of miR-663b inhibitor; inhibitor: cells transfected with miR-663b inhibitor; inhibitor+siTP73: cells co-transfected with miR-663b inhibitor and siTP73 RNA. All data are presented as the mean \pm SD of three independent experiments. $* *$ $\mathbf{p}<0.01$ vs control; \#\# $\mathbf{p}<0.01$ vs inhibitor. 
A

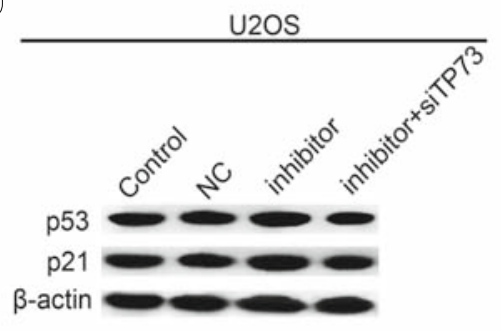

(B)

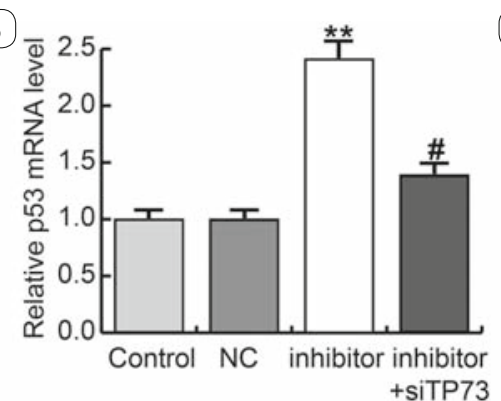

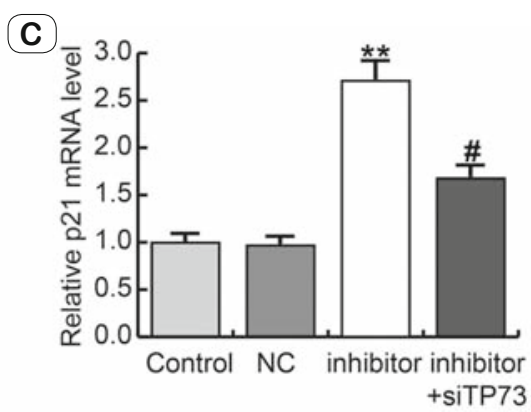

Fig. 6. p53 and p21 expression before and after miR-663b down-regulation. A: protein expression levels of p53 and p21 in U2OS cells; B and C: relative mRNA expression levels of p53 and p21 in U2OS cells. Control: cells without any treatment; NC: cells transfected with the negative control of miR-663b inhibitor; inhibitor: cells transfected with miR-663b inhibitor; inhibitor+siTP73: cells co-transfected with miR-663b inhibitor and siTP73 RNA. All data are presented as the mean \pm SD of three independent experiments. $* * \mathbf{p}<0.01$ vs control; \# $\mathbf{p}<0.05$ vs inhibitor.

miR-663b down-regulation promotes $p 53$ and $p 21$ expression

To explore the precise molecular mechanism of the effect of miR-663b on U2OS cell proliferation and apoptosis, the level of p53 and 211 was determined by using western blotting and qRTPCR analysis. As shown in Figure 6, compared with the control, miR-663b inhibitor significantly enhanced the expression of p53 and $\mathrm{p} 21$ at both the mRNA and the protein level, and the changes could be offset by siTP73 RNA.

\section{Discussion}

In the present study, we revealed that miR-663b down-regulation plays critical roles in preventing OS cell growth and inducing OS cell apoptosis. miR-663b directly targets TP73 and negatively regulates TP73 expression. miR-663b low-expression thus indirectly enhanced p53 and p 21 expression, which probably contributes to the inhibition of $\mathrm{U} 2 \mathrm{OS}$ cell proliferation and the induction of $\mathrm{U} 2 \mathrm{OS}$ cell apoptosis. First, we confirmed the high expression of miR-663b in osteosarcoma cell lines MG-63 and U2OS compared with the normal osteoblast hFOB1.19 cell line. And miR-663b expression is higher in U2OS cells than in MG-63 cells. Among the established human OS cell lines, U2OS cell line is widely used in scientific studies of OS (20). Therefore, in the present study, U2OS cell line was performed for the investigation of OS in vitro. We next found a novel functional link between miR-663b and TP73 in the progression of OS. The data also suggested miR-663b inhibitor significantly inhibited the proliferation and induced apoptosis of U2OS cells in vitro. p53 and p21 expression level was notably enhanced by miR-663b inhibition. Furthermore, we found that the effects of miR-663b inhibitor on U2OS cells could be reversed by siTP73 RNA. Our results revealed that miR-663b served as an oncogene in OS development, and miR-663b inhibitor plays important roles in tumor repression, thus, miR-663b may serve as a potential therapeutic target in the treatment of OS.

A growing number of studies suggest that miRNAs play an important role in cancer diagnosis, therapy and prognosis, and participate in tumorigenesis, tumor growth and tumor metastasis (10, 21). Thus, miRNAs may be potential targets for cancer therapy. A large number of studies reported that many miRNAs are involved in OS cell proliferation, migration and invasion (22-24). To date, the high expression of miR-663b in OS has been revealed however to the best of our knowledge the exact role of miR-663b in OS has not been investigated. Here, our results strongly proved that miR$663 \mathrm{~b}$ expression was significantly up-regulated in OS cell lines, and miR-663b inhibition significantly prevented the proliferation and induced apoptosis of U2OS cells.

TP73 (p73) is a member of the transcription factor p53 family and was first discovered in 1997. Due to its homology with the structure and function of $\mathrm{p} 53$, the research of $\mathrm{p} 73$ instead of $\mathrm{p} 53$ has become a hot spot. P73 can induce apoptosis and cell cycle arrest by binding to $\mathrm{p} 53$ response gene or trans activating $\mathrm{p} 53$ response gene (25). p73 has low expression in OS, and the triggering of $\mathrm{p} 73$ dependent apoptosis in OS is controlled by the E2Fs-pRb2/p130 complex $(26,27)$. However, no interaction between miR-663b and TP73 in OS cells has been reported previously. In the present study, we revealed that TP73 was a direct target of miR-663b, thus, miR-663b indirectly regulated the expression of $\mathrm{p} 53$ and $\mathrm{p} 21$.

In summary, we found for the first time that miR-663b downregulation inhibited the proliferation of OS cells and induced cell apoptosis via directly targeting TP73. Moreover, miR-663b inhibition could indirectly enhance the expression of p53 and p21. Therefore, miR-663b may serve as a potential therapeutic target for OS treatment.

\section{Reference}

1. Mirabello L, Troisi RJ, Savage SA: Osteosarcoma incidence and survival rates from 1973 to 2004: data from the Surveillance, Epidemiology, and End Results Program. Cancer 115: 1531-1543,2009.

2. Geller D S, Gorlick R: Osteosarcoma: a review of diagnosis, management, and treatment strategies. Clin Adv Hematol Oncol 2010; 8: 705-718.

3. Ottaviani G, Jaffe N. The epidemiology of osteosarcoma. Cancer Treat Res 2009; 152: 3-13.

4. Wang Q, Cai J, Wang J et al. miR-143 inhibits egfrsignaling-dependent osteosarcoma invasion. Tumour Biol 2014; 35: 12743-12748.

5. Liu Y, He J, Chen X et al. The proapoptotic effect of formononetin in human osteosarcoma cells: involvement of inactivation of erk and akt pathways. Cell Physiol Biochem 2014; 34: 637-645. 
$41-46$

6. Yang J, Zhang W. New molecular insights into osteosarcoma targeted therapy. Curr Opin Oncol 2013; 25: 398-406.

7. Tsuchiya H, Tomita K, Mori Y et al. Caffeine-assisted chemotherapy and minimized tumor excision for nonmetastatic osteosarcoma. Anticancer Res 1998; 18: 657-666.

8. Mei Q, Li X, Guo M,et al. The miRNA network: micro-regulator of cell signaling in cancer. Expert Rev Anticancer Ther 2014; 14: 1515-1527.

9. Ebert MS, Sharp PA. Roles for microRNAs in conferring robustness to biological processes. Cell 2012; 149: 515-524.

10. Rogers K, Chen X. Biogenesis, turnover, and mode of action of plant microRNAs. Plant Cell 2013; 25: 2383-2399.

11. Hayes J, Peruzzi PP, Lawler S. MicroRNAs in cancer: Biomarkers, functions and therapy. Trends Mol Med 2014; 20: 460-469.

12. Tsai MM, Wang CS, Tsai CY et al. Potential diagnostic, prognostic and therapeutic targets of microRNAs in human gastric cancer. Int J Mol Sci 2016; 17: 945.

13. Hu L, Ai J, Long $H$ et al. Intergrative microRNA and gene profiling data analysis reveals novel biomarkers and mechanisms for lung cancer. Oncotarget 2016; 7: 8441-8454.

14. Huafu Zhao, Mei Li, Lihua Li et al. MiR-133b Is Down-Regulated in Human Osteosarcoma and Inhibits Osteosarcoma Cells Proliferation, Migration and Invasion, and Promotes Apoptosis. PLoS One 2013; 8: e83571.

15. Xu L, Qi X, Duan S et al. MicroRNAs: potential biomarkers for disease diagnosis. Bio-Med Mater Eng 2014; 24: 3917-3925.

16. Zhang K, Zhang Y, Ren K et al. MicroRNA-101 inhibits the metastasis of osteosarcoma cells by downregulation of EZH2 expression. Oncol Rep 2014; 32: 2143-2149.

17. Yunlong Bi, Yu Jing, Yang Cao: Overexpression of miR-100 inhibits growth of osteosarcoma through FGFR3. Tumor Biol 2015; 36: 8405-8411.
18. Chen GN, Fang TT, Huang ZM et al. MicroRNA-133a Inhibits Osteosarcoma Cells Proliferation and Invasion via Targeting IGF-1R. Cell Physiol Biochem 2016; 38: 598-608.

19. Cheng DD, Yu T, Hu T et al. MiR-542-5p is a negative prognostic factor and promotes osteosarcoma tumorigenesis by targeting HUWE1. Oncotarget 2015; 6: 42761-42772.

20. Ponten J, Saksela E: Two established in vitro cell lines from human mesenchymal tumours. Int J Cancer 1967; 2: 434-447.

21. Cho WC. MicroRNAs: Potential biomarkers for cancer diagnosis, prognosis and targets for therapy. Int J Biochem Cell Biol 2010; 42: 1273 1281.

22. Luo XJ, Tang DG, Gao TL et al. MicroRNA-212 inhibits osteosarcoma cells proliferation and invasion by down-regulation of Sox4. Cell Physiol Biochem 2014; 34: 2180-2188.

23. Pan W, Wang H, Jianwei R et al. MicroRNA-27a promotes proliferation, migration and invasion by targeting MAP2K4 in human osteosarcoma cells. Cell Physiol Biochem 2014; 33: 402-412.

24. Chen B, Huang Z, Zhang Y et al. MicroRNA-145 Suppresses Osteosarcoma Metastasis via Targeting MMP16. Cell Physiol Biochem 2015; 37: 2183-2193.

25. Casciano I, Mazzocco K, Boni L et al. Expression of DeltaNp73 is a molecular marker for adverse outcome in neuroblastoma patients. Cell Death Differ2002; 9: 246-251.

26. Park HR, Kim YW, Park JH et al. Low expression of p63 and p73 in osteosarcoma. Tumori 2004; 90: 239-243.

27. La Sala D, Macaluso M, Trimarchi C et al. Triggering of p73-dependent apoptosis in osteosarcoma is under the control of E2Fs-pRb2/p130 complexes. Oncogene 2003; 22: 3518-3529.

Received August 2, 2017. Accepted August 25, 2017. 